\title{
Positive solutions for a one-dimensional Sturm-Liouville semipositone superlinear $p$-Laplacian problem
}

\author{
K. D. $\mathrm{Chu}^{1, *}$ and D. D. $\mathrm{Hai}^{2, * *}$ \\ ${ }^{1}$ Faculty of Mathematics and Statistics, Ton Duc Thang University, Ho chi Minh City, Vietnam \\ ${ }^{2}$ Department of Mathematics and Statistics, Mississippi state University, Mississippi State, MS 39762, \\ USA
}

Abstract. We prove the existence of a positive classical solution for the $p$ Laplacian equation

$$
-\left(r(t) \phi\left(u^{\prime}\right)\right)^{\prime}=-\lambda h(u)+f(t, u), t \in(0,1)
$$

with Sturm-Liouville boundary conditions, where $\phi(s)=|s|^{p-2} s, p>1, r$ : $[0,1] \rightarrow(0, \infty), f:(0,1) \times[0, \infty) \rightarrow \mathbb{R}$ is a Carathéodory function satisfying a superlinear condition at 0 and $\infty$ involving the principal eigenvalue of $-\left(r(t) \phi\left(u^{\prime}\right)\right)^{\prime}, h:(0, \infty) \rightarrow(0, \infty)$ is allowed to have infinite semipositone structure at 0 , and $\lambda \geq 0$ is a small parameter.

\section{Introduction}

Consider the one-dimensional $p$-Laplacian problem

$$
\left\{\begin{array}{c}
-\left(r(t) \phi\left(u^{\prime}\right)\right)^{\prime}=-\lambda g(u)+f(t, u), t \in(0,1), \\
a u(0)-b \phi^{-1}(r(0)) u^{\prime}(0)=0, c u(1)+d \phi^{-1}(r(1)) u^{\prime}(1)=0,
\end{array}\right.
$$

where $\phi(s)=|s|^{p-2} s, p>1, a, b, c, d$ are nonnegative constants with $\delta<1, a c+a d+b c>$ $0, r:[0,1] \rightarrow(0, \infty), f:(0,1) \times[0, \infty) \rightarrow \mathbb{R}, g:(0, \infty) \rightarrow(0, \infty)$, and $\lambda$ is a nonnegative parameter.

We are interested in positive classical solution of (1.1) in the superlinear case involving with the principal eigenvalue of the corresponding operator.

When $\lambda=0$ and $r \equiv 1$, Manásevich, Njoku, and Zanolin [14] used time-mapping estimates to prove the existence of a classical positive solution to (1.1) under Dirichlet boundary conditions when $f(t, z)$ satisfies

$$
\limsup _{z \rightarrow 0^{+}} \frac{f(t, z)}{z^{p-1}}<\lambda_{1}<\liminf _{z \rightarrow \infty} \frac{f(t, z)}{z^{p-1}} \leq \infty
$$

and $\liminf _{z \rightarrow 0^{+}} \frac{f(t, z)}{z^{p-1}}>-\infty$ uniformly for a.e. $t \in(0,1)$, where $\lambda_{1}=2^{p}(p-1)\left(\int_{0}^{1} \frac{d s}{\left(1-s^{p}\right)^{1 / p}}\right)^{p}$ is the principal eigenvalue of $-\left(\phi\left(u^{\prime}\right)\right)^{\prime}$ with zero boundary conditions (see [6,7]). Their result improved a previous work by Kaper, Knapp, and Kwong [12] where the stronger condition

$$
\lim _{z \rightarrow 0^{+}} \frac{f(t, z)}{z^{p-1}}=l \leq 0 \text { and } \lim _{z \rightarrow \infty} \frac{f(t, z)}{z^{p-1}}=\infty
$$

\footnotetext{
*e-mail: chuduckhanh@tdt.edu.vn

**e-mail: dang@math.msstate.edu
} 
uniformly for $t \in(0,1)$ had been used. Related results were obtained by Webb and Lan [15] when $p=2$, in which they gave a general method that covered many boundary conditions including nonlocal ones for both sublinear and superlinear cases involving with the principal eigenvalue of an operator. In the PDE case, the existence of a positive solution to the problem

$$
-\Delta u=f(u) \text { in } \Omega, u=0 \text { on } \partial \Omega
$$

with $f \geq 0$ satisfying (1.2) was obtained in [10], while similar results for the $p$-Laplacian

$$
-\Delta_{p} u=f(u) \text { in } \Omega, u=0 \text { on } \partial \Omega
$$

with $p>1$ were obtained in [5] using Granas fixed point index [8]. The existence result in [14] was generalized to the Sturm-Liouville boundary value problem (1.1) with $\lambda=0$ in [3] and to the singular problem (1.1) with $\lambda \geq 0$ small and $g(u)=u^{-\delta}$ for some $\delta \in[0,1)$ under Dirichlet boundary condition in [4]. In this note, we shall extend the result in [4] to the Sturm-Liouville boundary condition with a more general $g(u)$ allowing the challenging infinite semipositone case i.e. $\lim _{u \rightarrow 0^{+}} g(u)=-\infty$ (see [13]). We refer to $[9,11,16]$ and the references therein for the literature on existence results in the superlinear/sublinear cases not involving with the principal eigenvalue of the corresponding operator.

Let $\lambda_{1}$ be the principal eigenvalue of $-\left(r(t) \phi\left(u^{\prime}\right)\right)^{\prime}$ on $(0,1)$ with Sturm-Liouville boundary condition in (1.1) and let $\phi_{1}>0$ be the corresponding eigenfunction with $\left\|\phi_{1}\right\|_{\infty}=1$ (see [2]).

We shall assume the following conditions:

(A1) $r:[0,1] \rightarrow(0, \infty)$ is continuous and $g:(0, \infty) \rightarrow(0, \infty)$ is continuous, integrable on $(0,1)$ and decreasing.

(A2) $f:(0,1) \times[0, \infty)$ is a Carathéodory function, that is $f(., z)$ is measurable for each $z \geq 0$ and $f(t,$.$) is continuous for a.e. t \in(0,1)$.

(A3) For each $k>0$, there exists $\gamma_{k} \in L^{1}(0,1)$ such that

$$
|f(t, z)| \leq \gamma_{k}(t)
$$

for a.e. $t \in(0,1)$ and all $z \in[0, k]$.

(A4) There exists $\gamma \in L^{1}(0,1)$ with $\gamma \geq 0$ such that

$$
f(t, z)+\gamma(t) z^{p-1} \geq 0
$$

for a.e. $t \in(0,1)$ and all $z \geq 0$.

(A5) There exists a subset $B \subset[0,1]$ of measure 0 such that

$$
\limsup _{z \rightarrow 0^{+}} \frac{f(t, z)}{z^{p-1}}<\lambda_{1}<\liminf _{z \rightarrow \infty} \frac{f(t, z)}{z^{p-1}}
$$

uniformly for $t \in[0,1] \backslash B$.

By a positive classical solution of (1.1), we mean a function $u \in C^{1}[0,1]$ with $u>0$ on $(0,1), r(t) \phi\left(u^{\prime}\right)$ absolutely continuous on $[0,1]$ and satisfying $(1.1)$.

Our main result is

Theorem 1.1. Let (A1)-(A5) hold. Then there exists a constant $\lambda_{0}>0$ such that for $\lambda<$ $\lambda_{0},(1.1)$ has a positive classical solution $u$ with $\inf _{(0,1)} \frac{u}{\omega}>0$, where $\omega(t)=\min (a t+b, d+c(1-t))$.

In particular, when $f$ is independent of $t$, we obtain 
Corollary 1.1. Let $r$ satisfy $(A 1)$ and let $f:[0, \infty) \rightarrow R$ be continuous with

$$
-\infty<\lim _{z \rightarrow 0^{+}} \frac{f(z)}{z^{p-1}}<\lambda_{1}<\lim _{z \rightarrow \infty} \frac{f(z)}{z^{p-1}} \leq \infty .
$$

Then there exists a constant $\lambda_{0}>0$ such that for $\lambda<\lambda_{0},(1.1)$ has a positive classical solution $u$ with $\inf _{(0,1)} \frac{u}{p}>0$

\section{Preliminary results}

We shall denote the norm in $L^{q}(0,1)$ and $C^{1}[0,1]$ by $\|.\|_{q}$ and $|\cdot|_{C^{1}}$ respectively. We recall the following fixed point theorem of Krasnoselskii type in a Banach space (see Amann [1, Theorem 12.3]).

Theorem A. [1, Theorem 12.3] Let E be a Banach space and A $: E \rightarrow$ E be a completely continuous operator. Suppose there exist $h \in E, h \neq 0$ and positive constants $r, R$ with $r \neq R$ such that

(a) If $y \in E$ satisfies $y=\theta$ Ay for some $\theta \in(0,1]$ then $\|y\| \neq r$,

(b) If $y \in E$ satisfies $y=A y+\xi h$ for some $\xi \geq 0$ then $\|y\| \neq R$.

Then $A$ has a fixed point $y \in E$ with $\min (r, R)<\|y\|<\max (r, R)$.

Lemma 2.1. Let $\gamma, h \in L^{1}(0,1)$ with $\gamma, h \geq 0$ and let $\lambda>0, u \in C^{1}[0,1]$ satisfy

$$
\left\{\begin{array}{c}
-\left(r(t)\left(\phi\left(u^{\prime}\right)\right)^{\prime}+\gamma(t) \phi(u) \geq-\lambda h \text { on }(0,1),\right. \\
a u(0)-b \phi^{-1}(r(0)) u^{\prime}(0) \geq 0, c u(1)+d \phi^{-1}(r(1)) u^{\prime}(1) \geq 0 .
\end{array}\right.
$$

Then there exist constants $\kappa, \sigma>0$ independent of $u, \lambda, h$ such that

$$
u(t) \geq\left(\kappa\|u\|_{\infty}-\sigma\left(\lambda\|h\|_{1}\right)^{\frac{1}{p-1}}\right) p(t)
$$

for $t \in[0,1]$.

Proof. Let $v \in C^{1}[0,1]$ satisfy

$$
\left\{\begin{array}{c}
-\left(r(t)\left(\phi\left(v^{\prime}\right)\right)^{\prime}+\gamma(t) \phi(v)=-\lambda h \text { on }(0,1)\right. \\
a v(0)-b \phi^{-1}(r(0)) v^{\prime}(0)=0, c v(1)+d \phi^{-1}(r(1)) v^{\prime}(1)=0 .
\end{array}\right.
$$

By [3, Lemma 2.4], $v \leq 0$ and $|v|_{C^{1}} \leq M\left(\lambda\|h\|_{1}\right)^{\frac{1}{p-1}}$, where $M>0$ is independent of $u$ and $\lambda$. By [3, Lemma 2.5], $u \geq v$ on [0,1]. Since there exists a constant $k_{0}>0$ such that $|v(t)| \leq k_{0}|v|_{C^{1}} \omega(t)$ for all $t \in[0,1]$ and $v \in C^{1}[0,1]$ satisfying the Sturm-Liouville boundary conditions in (1.1), it follows that $v(t) \geq-M k_{0}\left(\lambda\|h\|_{1}\right)^{\frac{1}{p-1}} \omega(t)$ for $t \in[0,1]$. If $\|u\|_{\infty} \leq C\left(\lambda\|h\|_{1}\right)^{\frac{1}{p-1}}$ for some $C>0$ then

$$
u(t) \geq-M k_{0}\left(\lambda\|h\|_{1}\right)^{\frac{1}{p-1}} \omega(t) \geq\left(\|u\|_{\infty}-\left(C+M k_{0}\right)\left(\lambda\|h\|_{1}\right)^{\frac{1}{p-1}}\right) \omega(t)
$$

for $t \in\left[0,1\right.$. Suppose $\|u\|_{\infty}>C\left(\lambda\|h\|_{1}\right)^{\frac{1}{p-1}}$, where $C>M$. Let $\|u\|_{\infty}=|u(\tau)|$ for some $\tau \in$ $[0,1]$. Then $u(\tau)>0$ for otherwise $\|u\|_{\infty}=-u(\tau) \leq-v(\tau) \leq M\left(\lambda\|h\|_{1}\right)^{\frac{1}{p-1}}$, a contradiction.

Let $z \in C^{1}[0, \tau]$ be the solution of

$$
\left\{\begin{array}{c}
-\left(r(t) \phi\left(z^{\prime}\right)\right)^{\prime}+\gamma(t) \phi(z)=-\lambda h \text { on }(0, \tau) \\
a z(0)-b \phi^{-1}(r(0)) z^{\prime}(0)=0, \quad z(\tau)=\|u\|_{\infty}
\end{array}\right.
$$


Note that $u \geq z \geq v \geq-M\left(\lambda\|h\|_{1}\right)^{\frac{1}{p-1}}$ on $[0, \tau]$. Let $z_{1}(t)=z(t)+M\left(\lambda\|h\|_{1}\right)^{\frac{1}{p-1}}$. Then $z_{1} \geq 0$ on $[0,1]$ and

$$
z_{1}(t)=z_{1}(0)+\int_{0}^{t} \phi^{-1}\left(\frac{r(0) \phi\left(z_{1}^{\prime}(0)+\int_{0}^{s}(\gamma(\xi) \phi(z)+\lambda h) d \xi\right.}{r(s)}\right) .
$$

If $b=0$ then $z(0)=v(0)=0$ and so $z_{1}^{\prime}(0)=z^{\prime}(0) \geq v^{\prime}(0) \geq-M\left(\lambda\|h\|_{1}\right)^{\frac{1}{p-1}}$. On the other hand, if $b \neq 0$ then $z_{1}^{\prime}(0)=\frac{a}{b \phi^{-1}(r(0))} z(0) \geq-\frac{M a}{b \phi^{-1}(r(0))}\left(\lambda\|h\|_{1}\right)^{\frac{1}{p-1}}$. Hence

$$
z_{1}^{\prime}(0) \geq-M_{1}\left(\lambda\|h\|_{1}\right)^{\frac{1}{p-1}}
$$

where $M_{1}=M$ if $b=0$ and $M_{1}=\frac{M a}{b \phi^{-1}(r(0))}$ if $b \neq 0$. Since $\phi\left(z_{1}^{\prime}(0)\right) \leq \phi\left(z_{1}^{\prime}(0)+M_{1}\left(\lambda\|h\|_{1}\right)^{\frac{1}{p-1}}\right)$ and

$$
(x+y)^{q} \leq 2^{(q-1)^{+}}\left(x^{q}+y^{q}\right) \text { for } x, y \geq 0, q>0,
$$

it follows from (2.2) that

$$
z_{1}(t) \leq m_{1}\left(z_{1}(0)+z_{1}^{\prime}(0)+M_{1}\left(\lambda\|h\|_{1}\right)^{\frac{1}{p-1}}+\phi^{-1}\left(\int_{0}^{t}\left(\gamma(\xi) \phi\left(z_{1}\right)+\lambda h\right) d \xi\right)\right),
$$

where $m_{1}>0$ is a constant depending only on $p$ and $r$. Hence using (2.4) again and the inequality $\phi(x)+\phi(y) \leq 2 \phi(x+y)$ for $x, y \geq 0$, we deduce that

$$
\begin{aligned}
\phi\left(z_{1}(t)\right) & \leq m_{2}\left(\phi\left(z_{1}(0)+z_{1}^{\prime}(0)+M_{1}\left(\lambda\|h\|_{1}\right)^{\frac{1}{p-1}}\right)+\lambda\|h\|_{1}+\int_{0}^{t} \gamma(s) \phi\left(z_{1}\right) d s\right) \\
& \leq 2 m_{2} \phi\left(z_{1}(0)+z_{1}^{\prime}(0)+M_{2}\left(\lambda\|h\|_{1}\right)^{\frac{1}{p-1}}\right)+m_{2} \int_{0}^{t} \gamma(s) \phi\left(z_{1}\right) d s
\end{aligned}
$$

for $t \in[0, \tau]$, where $M_{2}=M_{1}+1$ and $m_{2}=2^{(p-2)^{+}} \phi\left(m_{1}\right)$.

By Gronwall's inequality,

$$
\phi\left(z_{1}(t)\right) \leq 2 m_{2} \phi\left(z_{1}(0)+z_{1}^{\prime}(0)+M_{2}\left(\lambda\|h\|_{1}\right)^{\frac{1}{p-1}}\right) e^{m_{2} \int_{0}^{t} \gamma(s) d s}
$$

for $t \in[0, \tau]$. In particular when $t=\tau$, we obtain

$$
z(0)+z^{\prime}(0)+\left(M_{2}+M\right)\left(\lambda\|h\|_{1}\right)^{\frac{1}{p-1}} \geq \kappa_{0}\|u\|_{\infty},
$$

where $\kappa_{0}=\left(e^{-m_{2}\|\gamma\|_{1}} / 2 m_{2}\right)^{1 /(p-1)}$. Since

$$
\left(r(t) \phi\left(z^{\prime}\right)\right)^{\prime}=\gamma(t) \phi(z)+\lambda h \geq-\gamma(t) \lambda M^{p-1}\|h\|_{1} \text { on }[0, \tau],
$$

it follows upon integrating on $[0, t]$ that

$$
r(t) \phi\left(z^{\prime}(t)\right) \geq r(0) \phi\left(z^{\prime}(0)\right)-\lambda M_{3}\|h\|_{1},
$$

where $M_{3}=M^{p-1}\|\gamma\|_{1}$, which implies

$$
z^{\prime}(t) \geq \phi^{-1}\left(\frac{r(0) \phi\left(z^{\prime}(0)\right)-\lambda M_{3}\|h\|_{1}}{r(t)}\right)
$$


for $t \in[0, \tau]$. Using the inequality $\psi(x-y) \geq c_{1} \psi(x)-c_{2} \psi(y)$ for $x, y \geq 0$ and $\psi \in\left\{\phi, \phi^{-1}\right\}$, where $c_{1}=\psi(1 / 2), c_{2}=2 \psi(2)$, we deduce from (2.3) and (2.6) that

$$
z^{\prime}(t) \geq M_{4} z^{\prime}(0)-M_{5}\left(\lambda\|h\|_{1}\right)^{\frac{1}{p-1}} \geq-M_{6}\left(\lambda\|h\|_{1}\right)^{\frac{1}{p-1}}
$$

where $M_{4}, M_{5}$ are positive constants depending only on $p$ and $r$, and $M_{6}=M_{1} M_{4}+M_{5}$. If $b=0$ then $z(0)=0$ and (2.5) and (2.7) give

$$
\begin{gathered}
z(t)=\int_{0}^{t} z^{\prime} \geq\left(M_{4}\left(\left(\kappa_{0}\|u\|_{\infty}-\left(M+M_{2}\right)\left(\lambda\|h\|_{1}\right)^{\frac{1}{p-1}}\right)-M_{5}\left(\lambda\|h\|_{1}\right)^{\frac{1}{p-1}}\right) t\right. \\
=\left(\kappa_{1}\|u\|_{\infty}-\sigma_{1}\left(\lambda\|h\|_{1}\right)^{\frac{1}{p-1}}\right)(a t+b)
\end{gathered}
$$

for $t \in[0, \tau]$, where $\kappa_{1}=a^{-1} M_{4} \kappa_{0}, \sigma_{1}=a^{-1}\left(M_{4}\left(M+M_{2}\right)+M_{5}\right)$. On the other hand, if $b>0$ then $z^{\prime}(0)=\frac{a}{b \phi^{-1}(r(0))} z(0)$ from which $(2.5)$ becomes

$$
z(0) \geq \tilde{\kappa}_{1}\|u\|_{\infty}-M_{7}\left(\lambda\|h\|_{1}\right)^{\frac{1}{p-1}}
$$

where $\tilde{\kappa}_{1}=\kappa_{0}\left(1+\frac{a}{b \phi^{-1}(r(0))}\right)^{-1}, M_{7}=\left(M+M_{2}\right)\left(1+\frac{a}{b \phi^{-1}(r(0))}\right)^{-1}$. Hence it follows upon integrating (2.7) that

$$
\begin{aligned}
& z(t) \geq z(0)- M_{6}\left(\lambda\|h\|_{1}\right)^{\frac{1}{p-1}} t \geq \tilde{\kappa}_{1}\|u\|_{\infty}-\left(M_{6}+M_{7}\right)\left(\lambda\|h\|_{1}\right)^{\frac{1}{p-1}} \\
& \geq\left(\kappa_{1}\|u\|_{\infty}-\sigma_{1}\left(\lambda\|h\|_{1}\right)^{\frac{1}{p-1}}\right)(a t+b)
\end{aligned}
$$

for $t \in[0, \tau]$, where $\kappa_{1}=\frac{\tilde{\kappa}_{1}}{a+b}, \sigma_{1}=\frac{M_{6}+M_{7}}{b}$. Combining (2.8) and (2.9), we see that in any case there exist constants $\kappa_{1}$ and $\sigma_{1}>0$ independent of $u, z, \lambda, h$ such that

$$
u(t) \geq z(t) \geq\left(\kappa_{1}\|u\|_{\infty}-\sigma_{1}\left(\lambda\|h\|_{1}\right)^{\frac{1}{p-1}}\right)(a t+b)
$$

for $t \in[0, \tau]$. Next, let $w \in C^{1}[\tau, 1]$ be the unique solution of

$$
\left\{\begin{array}{c}
-\left(r(t) \phi\left(w^{\prime}\right)\right)^{\prime}+\gamma(t) \phi(w)=-\lambda h \text { on }(\tau, 1) \\
w(\tau)=\|u\|_{\infty}, \quad c w(1)+d \phi^{-1}(r(1)) w^{\prime}(1)=0
\end{array}\right.
$$

Then $u \geq w \geq v$ on $[\tau, 1]$. Let $w_{1}=w+M\left(\lambda\|h\|_{1}\right)^{\frac{1}{p-1}}$. Then $w_{1} \geq 0$ and by using the integral formula

$$
w_{1}(t)=w_{1}(1)+\int_{t}^{1} \phi^{-1}\left(\frac{-r(1) \phi\left(w_{1}^{\prime}(1)+\int_{s}^{1}(\gamma(\xi) \phi(w)+\lambda h) d \xi\right.}{r(s)}\right) d s
$$

for $t \in[\tau, 1]$ with the same arguments as above, we obtain

$$
u(t) \geq w(t) \geq\left(\kappa_{2}\|u\|_{\infty}-\sigma_{2}\left(\lambda\|h\|_{1}\right)^{\frac{1}{p-1}}\right)(d+c(1-t))
$$

for $t \in[\tau, 1]$, where $\kappa_{2}>0$ is a constant independent of $u, w, \lambda, h$. Hence if $C>\max \left(\sigma_{1} / \kappa_{1}, \sigma_{2} / \kappa_{2}, M\right)$ then it follows from (2.1),(2.10), and (2.11) that $u(t) \geq$ $\left(\kappa\|u\|_{\infty}-\sigma\left(\lambda\|h\|_{1}\right)^{\frac{1}{p-1}}\right) \omega(t)$ for $t \in[0,1]$, where $\kappa=\min \left(\kappa_{1}, \kappa_{2}, 1\right), \sigma=\max \left(\sigma_{1}, \sigma_{2}, C+M k_{0}\right)$, which completes the proof of Lemma 2.1 .

\section{Proof of the main result}


Proof of Theorem 1.1. Since $\lim \sup _{z \rightarrow 0^{+}} \frac{f(t, z)}{z^{p-1}}<\lambda_{1}$, there exist constants $r, \bar{\lambda}>0$ with $\bar{\lambda}<\lambda_{1}$ such that

$$
f(t, z) \leq \bar{\lambda} z^{p-1}
$$

for $z \leq r$ and a.e. $t \in(0,1)$. Let $\kappa, \sigma$ be given by Lemma 2.1 with $h(t)=g\left(r_{0} \omega(t)\right)$, where $r_{0}=\kappa r / 2$, and let $\lambda>0$ be small enough so that $\sigma\left(\lambda\|h\|_{1}\right)^{\frac{1}{p-1}}<r_{0}$. For $v \in E=C[0,1]$, we have $0 \leq f(t,|v|)+\gamma(t)|v|^{p-1} \in L^{1}(0,1)$ in view of (A2) and (A4). Hence the problem

$$
\left\{\begin{array}{c}
-\left(r(t) \phi\left(u^{\prime}\right)\right)^{\prime}+\gamma(t) \phi(u)=-\lambda g\left(\max \left(v, r_{0} \omega\right)\right)+f(t,|v|)+\gamma(t)|v|^{p-1} \text { on }(0,1), \\
a u(0)-b \phi^{-1}(r(0)) u^{\prime}(0)=0, c u(1)+d \phi^{-1}(r(1)) u^{\prime}(1)=0
\end{array}\right.
$$

has a unique solution $u=A v \in C^{1}[0,1]$. Since $A=T_{0} \circ S_{0}$, where $S_{0}: C[0,1] \rightarrow L^{1}(0,1)$ is defined by $\left(S_{0} v\right)(t)=-\lambda g\left(\max \left(v, r_{0} \omega\right)\right)+f(t,|v|)+\gamma(t)|v|^{p-1}$ and $T_{0}$ is defined in Lemma A with $\alpha=\beta=0$, we see that $A: E \rightarrow E$ is completely continuous. We shall verify that

(i) $u=\theta A u, \theta \in(0,1] \Longrightarrow\|u\|_{\infty} \neq r$.

Indeed, let $u \in E$ satisfy $u=\theta A u$ for some $\theta \in(0,1]$ and suppose $\|u\|_{\infty}=r$. Then $u \in$ $C^{1}[0,1]$ and

$$
\begin{aligned}
-\left(r(t) \phi\left(u^{\prime}\right)\right)^{\prime}+\gamma(t) \phi(u) & =\theta^{p-1}\left(-\lambda g\left(\max \left(u, r_{0} \omega\right)\right)+f(t,|u|)+\gamma(t)|u|^{p-1}\right) \\
& \geq-\lambda h(t)
\end{aligned}
$$

on $(0,1)$, from which Lemma 2.1 gives

$$
u(t) \geq\left(\kappa\|u\|_{\infty}-\sigma\left(\lambda\|h\|_{1}\right)^{\frac{1}{p-1}}\right) \omega(t) \geq r_{0} \omega(t)>0
$$

for $t \in(0,1)$. Hence

$$
-\left(r(t) \phi\left(u^{\prime}\right)\right)^{\prime} \leq \theta^{p-1} f(t, u)
$$

on $(0,1)$. Let $\delta_{0}=\sup _{(0,1)} \frac{u}{\phi_{1}} \in(0, \infty)$. Then it follows from (3.1) and (3.3) that

$$
-\left(r(t) \phi\left(u^{\prime}\right)\right)^{\prime} \leq \bar{\lambda} u^{p-1} \leq \bar{\lambda} \delta_{0}^{p-1} \phi_{1}^{p-1} \text { on }(0,1),
$$

from which the weak comparison principle gives

$$
u \leq\left(\bar{\lambda} \delta_{0}^{p-1} / \lambda_{1}\right)^{\frac{1}{p-1}} \phi_{1}
$$

on $[0,1]$, a contradiction with the definition of $\delta_{0}$. Thus $\|u\|_{\infty} \neq r$ i.e. (i) holds.

Next, we claim that

(ii) There exists a constant $R>r$ such that $u=A u+\xi, \xi \geq 0 \Longrightarrow\|u\|_{\infty} \neq R$.

Let $u \in E$ satisfy $u=A u+\xi$ for some $\xi \in[0, \infty)$. Then $u-\xi=A u$ and therefore

$$
-\left(r(t) \phi\left(u^{\prime}\right)\right)^{\prime}+\gamma(t) \phi(u-\xi)=-\lambda g\left(\max \left(u, r_{0} \omega\right)\right)+f(t,|u|)+\gamma(t)|u|^{p-1}
$$

on $(0,1)$, which implies

$$
-\left(r(t) \phi\left(u^{\prime}\right)\right)^{\prime}+\gamma(t) \phi(u) \geq-\lambda h(t)
$$

on $(0,1)$. Since $\liminf _{z \rightarrow \infty} \frac{f(t, z)-\lambda g(z)}{z^{p-1}}>\lambda_{1}$ uniformly for a.e. $t \in(0,1)$, there exist positive constants $L, \tilde{\lambda}$ with $\tilde{\lambda}>\lambda_{1}$ such that $f(t, z)-\lambda g(z) \geq \tilde{\lambda} z^{p-1}$ for a.e. $t \in(0,1)$ and $z>L$.

For $z \leq L,(\mathrm{~A} 2)$ gives $f(t, z)-\lambda g\left(\max \left(z, r_{0} \omega(t)\right)\right) \geq-\tilde{\gamma}_{L}(t)$ for a.e. $t \in(0,1)$ and all $z \geq 0$, where $\tilde{\gamma}_{L}=\gamma_{L}+\lambda h$. Suppose $\|u\|_{\infty}=R>r$. Then

$$
u(t) \geq\left(\kappa R-\sigma\left(\lambda\|h\|_{1}\right)^{\frac{1}{p-1}}\right) p(t) \geq \frac{\kappa R}{2} \omega(t) \geq \frac{\kappa R c}{2} \phi_{1}(t)
$$


for $t \in[0,1]$ in view of Lemma 2.1, where $c=\inf _{(0,1)} \frac{\omega}{\phi_{1}}$. In particular, $u(t) \geq r_{0} \omega(t)$ for $t \in[0,1]$. Let $\delta_{1}=\inf _{(0,1)} \frac{u}{\phi_{1}}$. Then $\delta_{1} \geq 1$ if $R>2 / \kappa c$, which we assume. Hence

$$
-\left(r(t) \phi\left(u^{\prime}\right)\right)^{\prime} \geq f(t, u)-\lambda g(u) \geq\left\{\begin{array}{ll}
\tilde{\lambda}_{u^{p-1}} & \text { if } u>L \\
-\tilde{\gamma}_{L} & \text { if } u \leq L
\end{array} .\right.
$$

Since $u(t)>L$ if $\omega(t)>2 L / \kappa R$, it follows that

$$
-\left(r(t) \phi\left(\frac{u^{\prime}}{\delta_{1}}\right)\right)^{\prime} \geq\left\{\begin{array}{ll}
\tilde{\lambda} \phi_{1}^{p-1} & \text { in } D_{R}, \\
-\tilde{\gamma}_{L} & \text { in }(0,1) \backslash D_{R}
\end{array} \equiv h_{1 R},\right.
$$

where $D_{R}=\{t: \omega(t)>2 L / \kappa R\}$. Let $u_{1 R}, u_{2} \in C^{1}[0,1]$ satisfy

$$
-\left(r(t) \phi\left(u_{1 R}^{\prime}\right)\right)^{\prime}=h_{1 R} \text { on }(0,1)
$$

and

$$
-\left(r(t) \phi\left(u_{2}^{\prime}\right)\right)^{\prime}=\tilde{\lambda} \phi_{1}^{p-1} \equiv h_{2} \text { on }(0,1)
$$

with Sturm-Liouville boundary condition in (1.1). Note that $u_{2}=\left(\tilde{\lambda} / \lambda_{1}\right)^{\frac{1}{p-1}} \phi_{1}$. Since

$$
\left\|h_{1 R}-h_{2}\right\|_{1}=\int_{(0,1) \backslash D_{R}}\left(\tilde{\lambda} \phi_{1}^{p-1}+\tilde{\gamma}_{L}\right) \rightarrow 0
$$

as $R \rightarrow \infty$, it follows that $\left|u_{1 R}-u_{2}\right|_{C^{1}} \rightarrow 0$ as $R \rightarrow \infty$ (see e.g. [11, Lemma 3.1]). Let $\varepsilon>0$ be such that $\left(\tilde{\lambda} / \lambda_{1}\right)^{\frac{1}{p-1}}-\varepsilon c_{0} \equiv c_{1}>1$, where $c_{0}=\sup _{(0,1)} \frac{\omega}{\phi_{1}}>0$. Then, if $R$ is large enough (independent of $u$ ), we get

$$
u_{1 R} \geq u_{2}-\varepsilon \omega \geq u_{2}-\varepsilon c_{0} \phi_{1}=c_{1} \phi_{1} \text { on }(0,1),
$$

and consequently, $u \geq \delta_{1} u_{1 R} \geq \delta_{1} c_{1} \phi_{1}$ on $(0,1)$, a contradiction with the definition of $\delta_{1}$. Thus $\|u\|_{\infty} \neq R$ if $R$ is large enough i.e. (ii) holds.

By Lemma A, $A$ has a fixed point $u \in E$ with $\|u\|_{\infty}>r$, which is a classical positive solution of (1.1) in view of (3.2) and Lemmas 2.2. This completes the proof of Theorem 1.1.

\section{References}

[1] H. Amann, Fixed point equations and nonlinear eigenvalue problems in ordered Banach Spaces, SIAM Rev. 18 (1976), 620-709.

[2] P. Binding and P. Drabek, Sturm-Liouville theory for the p-Laplacian, Studia Sci. Math. Hunga. 40 (2003), no. 4, 375-396.

[3] K. D. Chu and D. D. Hai, Positive solutions for the one-dimensional Sturm-Liouville superlinear p-Laplacian problem, Electron. J. Differential Equations (2018), No. 92, pp. $1-14$.

[4] K. D. Chu and D. D. Hai, Positive solutions for the one-dimensional singular superlinear $p$-Laplacian (submitted).

[5] A. Cwiszewski and M. Maciejewski, Positive stationary solutions for $p$-Laplacian problems with nonpositive perturbation, J. Differential Equations 254 (2013), 1120-1136.

[6] M. Del Pino, M. Elgueta, and R. Manasevich, A homotopic deformation along $p$ of a Leray-Schauder degree result and existence for $\left(\left|u^{\prime}\right|^{p-2} u^{\prime}\right)^{\prime}+f(t, u)=0, u(0)=u(T)=$ 0, $p>1$. J. Differential Equations 80 (1989), no. 1, 1-13. 
[7] P. Drabek, Ranges of a -homogeneous operators and their perturbations, Casopis Pest. Mat. 105 (1980), 167-183.

[8] J. Dugundji and A. Granas, Fixed Point Theory, Springer-Verlag, 2004.

[9] L. Erbe and H. Wang, On the existence of positive solutions of ordinary differential equations. Proc. Amer. Math. Soc. 120 (1994), no. 3, 743-748.

[10] D. de Figueiredo, P. L. Lions, and R. D. Nusbaum, Estimations a priori pour les solutions positives de problèmes elliptiques superlinéaires. (French) C. R. Acad. Sci. Paris Sér. A-B 290 (1980), no. 5, 217-220.

[11] D. D. Hai, On singular Sturm-Liouville boundary-value problems. Proc. Roy. Soc. Edinburgh Sect. A 140 (2010), no. 1, 49-63.

[12] H. G. Kaper, M. Knaap, and M. K. Kwong, Existence theorems for second order boundary value problems. Differential Integral Equations 4 (1991), no. 3, 543-554.

[13] E. Lee, R. Shivaji, and J. Ye, Subsolutions: a journey from positone to infinite semipositone problems, Electron. J. Differ. Equ. Conf. 17 (2009), 123-131.

[14] R. Manásevich, F. Njoku and Zanolin, F, Positive solutions for the one-dimensional p-Laplacian. Differential Integral Equations 8 (1995), 213-222.

[15] J. R. L. Webb and K. Q. Lan, Eigenvalue criteria for existence of multiple positive solutions of nonlinear boundary vale problems of local and nonlocal types, Topol. Methods Nonlinear Anal. 27 (2006), 91-116.

[16] J. Wang, The existence of positive solutions for the one-dimensional $p$-Laplacian, Proc. Amer. Math. Soc. 125 (1997), 2275-2283. 